\title{
Introduction
}

\section{Cancer-associated thrombosis}

\author{
AK Kakkar*,I
'Thrombosis Research Institute and Queen Mary University of London, Emmanuel Kaye Building Manresa Road, London SW3 6LR, UK \\ British Journal of Cancer (2010) I 02, SI. doi:I0.I038/sj.bjc.6605598 www.bjcancer.com \\ (C) 2010 Cancer Research UK
}

Venous thromboembolism (VTE) is an important and potentially fatal complication in patients with malignant disease. Increasingly, VTE is recognised to affect not only patients undergoing surgical intervention for the management of their cancer but also those receiving chemotherapy, including ambulatory patients. The extent to which thrombosis complicates the natural history of different populations of patients with malignant disease is poorly understood. The risk factors associated with the development of thromboembolic complications include stage of disease, anatomical site of tumour, and tumour histology, in addition to commonly recognised risk factors for the development of thromboembolism, such as advanced stage, obesity, and a history of VTE.

The type of therapeutic intervention also has an important impact on the risk for VTE. It is well recognised that patients undergoing laparotomy for intra-abdominal or pelvic malignancy are at heightened risk for the development of postoperative VTE. For those receiving cytotoxic chemotherapy, the nature of cytotoxic therapy may influence risk for VTE. Increasingly, however, there is also recognition of the importance of biological anticancer therapies in heightening the risk for thrombosis. This multimodal interaction between patient and therapeutic factors makes identification of patients at risk for thrombosis difficult, but various methods, including risk-assessment scores and the development of biomarkers to predict those at risk for thrombosis, are currently under development.

It is well established that pharmacological thromboprophylaxis is warranted in the majority of patients with cancer undergoing surgery. At present, low-dose unfractionated heparin or lowmolecular-weight heparins (LMWHs) are the thromboprophylactic modalities of choice. In general, prophylaxis is usually provided for the duration of hospital stay, but - in some cases - may be extended for patients with an increased risk for thromboembolic disease after hospital discharge.

For patients undergoing medical management of their cancer and who are hospitalised with an acute medical condition, we recommended thromboprophylaxis with LMWHs for the duration of hospital stay. Considerable controversy exists regarding the benefits of extended prophylaxis on an outpatient basis for ambulant patients receiving chemotherapy, as guidelines currently do not recommend routine prophylaxis for this group. For those patients with established thromboembolism, long-term therapy with an LMWH offers important advantages in reducing the frequency of recurrent thromboembolic disease compared with those receiving standard anticoagulant therapy for the prevention of recurrent thromboembolism with a vitamin $\mathrm{K}$ antagonist.

Intriguingly, exposure to LMWHs has been suggested to prolong survival in patients with malignant disease. Early studies - many of which have had methodological flaws - suggested that LMWHs may indeed prolong survival in patients with cancer. Ongoing studies will provide further information regarding which patient populations benefit most from exposure to LMWHs in terms of survival.

This supplement provides a broad-based review of current topics in the prevention and treatment of VTE, as well as the impact of treatment with anticoagulants on prognosis and survival in patients with cancer, with particular focus on the management of patients in the United Kingdom. An expert panel was convened in London to review data related to the pathophysiology, epidemiology, prevention, and treatment of VTE, and the role of antithrombotic therapy in the survival of patients with cancer. The panel also reviewed international guidelines for the prophylaxis and treatment of VTE to assess and interpret this information in the context of current practices in the United Kingdom. Each article in this supplement consists of a data review based on the presentations given at the meeting, a transcript of the discussions held around each topic, and - finally - a brief consensus statement discussing treatment recommendations and research priorities for the future. These articles, when taken together, represent the state of the art in the treatment and prevention of VTE in cancer patients, and aim to improve clinical practice in the United Kingdom for this important patient population.

\section{ACKNOWLEDGEMENTS}

I thank John Ferguson and Thrombosis Education Ltd for providing medical writing services. Funding for this supplement was sponsored by an unrestricted donation from Pfizer Ltd. The content of the papers was provided by the authors and Pfizer had no input in the writing or approval of the content.

\section{Conflict of interest}

AK Kakkar has received consulting fees from Bayer, Sanofiaventis, Boehringer Ingelheim, Pfizer, Bristol-Myers Squibb, and Eisai.

\footnotetext{
*Correspondence: Professor AK Kakkar;

E-mail: akkakkar@tri-london.ac.uk
} 